\title{
Temporal and Spatial Expression of Distinct Troponin T Genes in Embryonic/Larval Tail Striated Muscle and Adult Body Wall Smooth Muscle of Ascidian
}

\author{
Takeshi Endo*, Ken Matsumoto, Taro Hama, Yukio Ohtsuka, Go Katsura, and Takashi Obinata \\ Department of Biology, Faculty of Science, Chiba University, Yayoicho, Inageku, Chiba, Chiba 263, Japan
}

Key words: troponin T/ascidian/smooth muscle/striated muscle/nuclear localization

\begin{abstract}
During development of the ascidian Halocynthia roretzi, the tadpole larva hatched from the tailbud embryo metamorphoses to the adult with a body wall muscle. Although the adult body wall muscle is morphologically nonsarcomeric smooth muscle, it contains a troponin complex consisting of three subunits (T, I, and C) as do vertebrate striated muscles. Different from vertebrate troponins, however, the smooth muscle troponin promotes actin-myosin interaction in the presence of high concentration of $\mathrm{Ca}^{2+}$, and this promoting property is attributable to troponin $T$. To address whether the embryonic/larval tail striated muscle and the adult smooth muscle utilize identical or different regulatory machinery, we cloned troponin T cDNAs from each cDNA library. The embryonic and the adult troponin Ts were encoded by distinct genes and shared only $<60 \%$ identity with each other. These isoforms were specifically expressed in the embryonic/larval tail striated muscle and the adult smooth muscle, respectively. These results may imply that these isoforms regulate actin-myosin interaction in different manners. The adult troponin $T$ under forced expression in mouse fibroblasts was unexpectedly located in the nuclei. However, a truncated protein with a deletion including a cluster of basic amino acids colocalized with tropomyosin on actin filaments. Thus, complex formation with troponin I and $\mathrm{C}$ immediately after the synthesis is likely to be essential for the protein to properly localize on the thin filaments.
\end{abstract}

Troponin (Tn) complex, which consists of three subunits $\mathrm{T}$, I and $\mathrm{C}$, is the $\mathrm{Ca}^{2+}$-dependent negative regulator of vertebrate striated muscle contraction (1). This protein complex is associated with tropomyosin $(\mathrm{Tm})$ on the thin filaments with a periodicity of $38 \mathrm{~nm}$. Tn does not exist in vertebrate smooth muscle, and its contraction is primarily regulated by $\mathrm{Ca}^{2+} /$ calmodulin-dependent phosphorylation of myosin light chain $(2,3)$. $\mathrm{Tn} / \mathrm{Tm}$-regulatory system also prevails in a variety of invertebrate striated and obliquely striated muscles (4). Tns in these invertebrate muscles also inhibit actin-myosin interaction dependently on $\mathrm{Ca}^{2+}$. The presence of Tn has not been demonstrated in smooth or nonstriated muscles except for the adult body wall muscle of the ascidian Halocynthia roretzi $(5,6)$, a protochordate. The ascidian body wall muscle is composed of multinucleated but nonsarcomeric smooth muscle cells (7). Differ-

\footnotetext{
* Correspondence should be addressed to Takeshi Endo, Department of Biology, Faculty of Science, Chiba University, 1-33 Yayoicho, Inageku, Chiba, Chiba 263, Japan.

Tel and Fax: +81-43-290-3911

e-mail: tendo@cuphd.nd.chiba-u.ac.jp

Abbreviations: $\mathrm{Tn}$, troponin; $\mathrm{TnT}$, troponin $\mathrm{T}$; TnI, troponin I; $\mathrm{TnC}$, troponin $\mathrm{C}$; $\mathrm{Tm}$, tropomyosin; PAGE, polyacrylamide gel electrophoresis; NLS, nuclear localization signal.
}

ent from Tns from the other sources, the ascidian Tn activates actin-myosin interaction in the presence of high concentration of $\mathrm{Ca}^{2+}(6,8)$. The activating property of ascidian $\mathrm{Tn}$ is ascribable to $\mathrm{TnT}$, because the isolated $\mathrm{TnT}$ promotes actin-myosin interaction regardless of $\mathrm{Ca}^{2+}$ concentration (6). The ascidian TnT shares with vertebrate counterparts some other properties including Tm-binding ability (6), basic pI, consisting of multiple isoelectric forms, and crossreactivity to the monoclonal antibody NT302 (9) raised against chicken skeletal muscle TnT (10).

During development of the ascidian, the tailbud embryo hatches out to generate the swimming tadpole larva, which metamorphoses to the sessile adult. The tails of the embryo and the larva contain mononucleated striated muscle cells or their precursor cells. A myosin heavy chain gene and an $\alpha$-actin gene are specifically expressed in the striated muscle cells and the precursor cells but not in the adult smooth muscle $(11,12)$. In contrast, a Tm gene is expressed in the adult body wall muscle as well as striated muscles of adult heart and larval tail in another ascidian Ciona intestinalis (13). As to Tn subunits, there has been no report regarding their expression at the embyonic or larval stage. Thus, it remains to be determined which type of regulatory system 
of muscle contraction dominates in the embryonic and larval striated muscles.

Determination of the primary and higher structures of the adult $H$. roretzi $\mathrm{TnT}$ and embryonic or larval counterparts, if present, may answer the questions how the adult $\mathrm{TnT}$ accelerates actin-myosin interaction, how its multiple isoelectric forms are generated, and how the embryonic or larval striated muscle contraction is regulated. To these ultimate ends, we cloned the TnT cDNAs by screening adult body wall muscle and tailbud embryo cDNA expression libraries with NT302. The embryonic and adult proteins encoded by these cDNAs were only $<60 \%$ identical to each other. In addition, they were specifically expressed in the embryonic/larval tail striated muscle and the adult smooth muscle, respectively, suggesting their functional difference. Forced expression of the adult TnT in cultured mammalian nonmuscle cells further suggest that complex formation with the other troponin subunits is required for the protein to associate with thin filaments or microfilaments in vivo.

\section{MATERIALS AND METHODS}

All the materials and methods used in this study are described elsewhere (14).

\section{RESULTS}

Sequence of TnT clones from adult body wall smooth muscle and tailbud embryo. To clone adult $H$. roretzi body wall smooth muscle TnT cDNAs, we exploited crossreactivity of the monoclonal antibody NT302 to TnTs from various vertebrate and protochordate animals including the ascidian TnT isoelectric forms (9). The body wall muscle $\lambda \mathrm{gt} 11 \mathrm{cDNA}$ expression library was screened with the antibody and overlapping three clones were isolated. The longest clone, aTnT2, was $923 \mathrm{bp}$ and contained an open reading frame encoding a 248-amino acid protein. To investigate whether similar but distinct TnT mRNAs exist in the body wall muscle, we further screened the $\lambda \mathrm{gt} 10$ cDNA library with aTnT2 and isolated $\sim 30$ clones. One of the longest clones, aTnT19, was $1,164 \mathrm{bp}$ and completely overlapped with aTnT2 between nucleotides 208 and 1130, where a common open reading frame existed (Fig. 1). aTnT19 contained additional $207 \mathrm{bp} \mathrm{nu-}$ cleotides upstream of the $5^{\prime}$ end of aTnT2 and additional $34 \mathrm{bp}$ nucleotides downstream of the $3^{\prime}$ end. A protein encoded by aTnT2 and aTnT19 consisted of 248 amino acids with a calculated molecular mass of 29,770 Da and a calculated pI of 9.56. These values were close to those estimated by SDS-PAGE (6) and two-dimensional isoelectric focusing/SDS-PAGE (9).

We next screened the tailbud embryo $\lambda \mathrm{gt} 11 \mathrm{cDNA}$ ex-

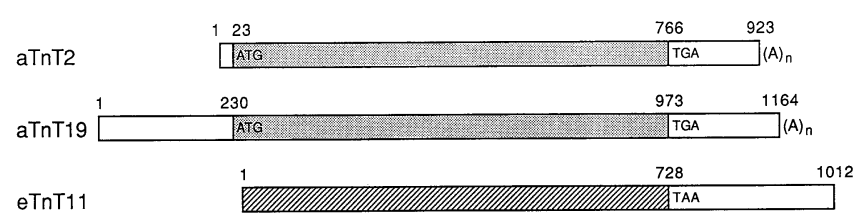

Fig. 1. Schematic representation of aTnT19, aTnT2, and eTnT11 cDNAs. Stippled and hatched areas represent TnT-encoding regions. The positions of the initiation and termination codons and poly(A) tails are indicated. Their nucleotide sequences and predicted amino acid sequences are reported by Endo et al. (14).

pression library with NT302 to determine which type of regulatory system governs in the tail striated muscle of $H$. roretzi tailbud embryo and tadpole larva. Two positive clones obtained (eTnT11 and 14) were 1,012-bp long and their nucleotide sequences were identical to each other (Fig. 1). Since no in-frame termination codon was present upstream of the $\mathrm{A}^{36} \mathrm{TG}$ in these clones, they may contain a partial coding sequence lacking the 5 -terminal sequence including the initiation codon. If this is true for these clones, they code for an $\mathrm{N}$-terminal-truncated protein consisting of 242 amino acids with a calculated molecular mass of $28,615 \mathrm{Da}$ and a calculated pI of 10.22 . The identity of sequence between aTnT2/aTnT19 and eTnT11/eTnT14 was only $\sim 60 \%$, and identical sequences were extremely dispersed. This implies that they were derived from different genes.

Comparison of the amino acid sequence among ascidian and vertebrate TnTs. The amino acid sequences of the proteins encoded by aTnT2/aTnT19 (hereafter the protein is referred to as adTnT) and by eTnT11 (the protein is designated as embTnT) were compared with each other and with those of chicken skeletal (15) and cardiac (16) and rabbit skeletal (17) and cardiac (18) muscle TnTs. adTnT and embTnT were only $58.9 \%$ identical to each other, but they were still more similar to each other than to vertebrate TnTs. In addition, it is worth noting that the identity between skeletal and cardiac muscle TnTs is only 54 and 56\% in chicken and rabbit, respectively.

Analyses of cyanogen bromide- or chymotrypsindigested fragments of rabbit skeletal muscle TnT have shown that $\mathrm{Tm}$ - and TnI-binding abilities reside in Glu $^{71}{ }_{-}$Ser $^{150}$ and Lys $^{223}-\mathrm{Tyr}^{227}$, respectively (19). When the amino acid sequences of adTnT and embTnT were aligned with those of the rabbit skeletal and cardiac muscle TnTs to search similarity according to Lipman and Pearson (20), central regions were more conserved than the N- and C-terminal regions (Fig. 2). The above $\mathrm{Tm}$ - and TnI-binding sites were located in these conserved areas. Adult $H$. roretzi TnT binds to Tm as determined by electron microscopic observations of the binding to Tm paracrystals (6). In addition, the fact that the 
Ascidian Smooth and Striated Muscle Troponin Ts

$\begin{array}{lrrr} & 1 & \text { SDLEEVVEEYEEEQEEQEAGEEEEAGG } & 27 \\ \text { Rab (Card) } & 28 & \text { GAEAEAETEETQAEEDGQEEEDKEDEDGPVEESKPKPRPFMPNLVPPK IPDGERVDFDDI } & 87 \\ \text { adTnT } & 1 & \text { MSGEEEEPQQHHEDVEKPMPRHSTTSI-DPRPDGEKVDLDVI } & 41 \\ \text { embTnT } & 1 & \text { DSTGKVAPKFTMPNITPPKIPDGEVIDUEDI } & 31 \\ \text { Rab (Sk) } & 1 & \text { SDEEVEHVEEEAEEEAPSPAEVHEPAPEHVVPEEVHEEEKPRKLTAPKIPEGEKVDFDDI } & 60\end{array}$

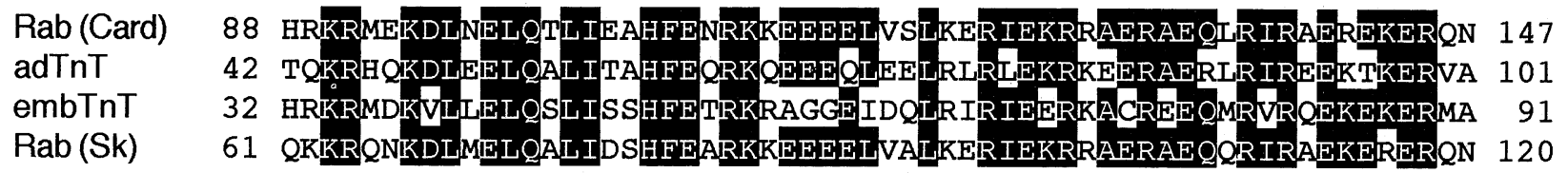

Tm-binding

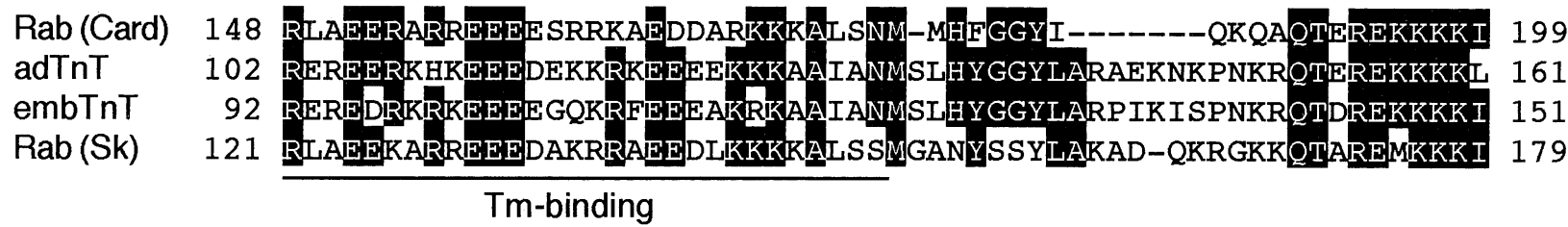

Rab (Card) 200 LAERRKVLAIDHLNEDQLREKAKELWQSIYNLEAEKFDLQEKFKOQKYEINVLRNRIND- 258 adTnT 162 LAERKKNISVDHLSPDKLREKAQELWDLLYSLEEEK IDYEVRIDRQK YDLNQLRQRVNEY 221 embTnT 152 LSDRRKPLNVDHLSAEKLQEKASELWKWLYSLEEEKYDFETRIERQKYDINQLRQRVNEY 211 Rab (Sk) 180 LAERRKPLNIDHLSDEKLRDKAKELWDTLYQLETDKFEFGEKLKROKYD IMNVRARV-EM 238

Tn I-binding

$\begin{array}{llll}\text { Rab (Card) } & 259 & \text { NQRVISKTR----GKA---KVTGRWK } & 276 \\ \text { adTnT } & 222 \text { MGKYSKNK----SKVKVAGHGGVMIKAASAFK } & 248 \\ \text { embTnT } & 212 \text { MGKFSKTKRQTPGKVNTGARGGVSSKTGVFS } & 242 \\ \text { Rab(Sk) } & 239 \text { LAKFSKKA----GTTAKGKVGGRWK } & 259\end{array}$

Fig. 2. Comparison of the amino acid sequences of adTnT and embTnT with those of rabbit skeletal and cardiac muscle TnTs. Amino acid sequences of adTnT, embTnT, and rabbit skeletal and cardiac muscle TnTs were aligned to search similarity by the method of Lipman and Pearson (20). Insertion made during optimization is marked with a dash. Amino acids at positions of $\geq 75 \%$ identity are shown in white on black. Tmand TnI-binding sites of rabbit skeletal muscle TnT are marked by single and dotted underlines, respectively.

ability of adult TnT to accelerate actin-myosin interaction is inhibited by TnI (6) suggests that the TnT interacts with TnI. The identity between adTnT and embTnT in the corresponding areas to Tm-binding and TnI-binding was 60.0 and $80.0 \%$, respectively, which was higher than the identity in the whole length of the molecules. This may indicate that embTnT also has abilities to interact with Tm and TnI, implying that it functions as TnT.

In rabbit skeletal muscle TnT, $\operatorname{Ser}^{1}$ is acetylated and phosphorylatable by casein kinase $(17,19)$. $\operatorname{Ser}^{149}$ or $\operatorname{Ser}^{150}$ and $\operatorname{Ser}^{156}$ or $\operatorname{Ser}^{157}$ are also phosphorylatable by phosphorylase kinase (19). Existence of these multiple phosphorylation sites in addition to isoforms produced by alternative splicing may account for multiple isoelectric variants of $\operatorname{TnT}(9,10)$. If $\operatorname{Met}^{1}$ in adTnT is removed by a methionine-specific aminopeptidase, the first residue is Ser as in rabbit skeletal and cardiac mus- cle TnTs, and this Ser might also be acetylated and phosphorylated. In this context, it should be noted that this Ser, as well as Ser in rabbit skeletal and cardiac muscle TnTs, fits in the consensus phosphorylation site by casein kinase II [(S/T)XX(D/E)] (21) (Fig. 2). Residues corresponding to Ser ${ }^{149 / 150}$ and $\mathrm{Ser}^{156 / 157}$ in rabbit skeletal muscle TnT were, however, replaced by other amino acids in adTnT and embTnT.

Existence of embTnT in the embryonic/larval tail striated muscles. In Northern blotting, aTnT2 hybridized to the adult body wall muscle mRNA apparently as a single broad band of $\sim 1.1 \mathrm{~kb}$ but neither to the larval mRNA nor to the vertebrate muscle mRNAs examined (Fig. 3). eTnT11 hybridized to the larval mRNA as doublet bands of $\sim 1.6$ and $\sim 1.4 \mathrm{~kb}$ but not to the adult body wall muscle or vertebrate muscle mRNAs (Fig. 3). These doublet bands might correspond to mRNAs generated by alternative $3^{\prime}$ end processing because the differ- 


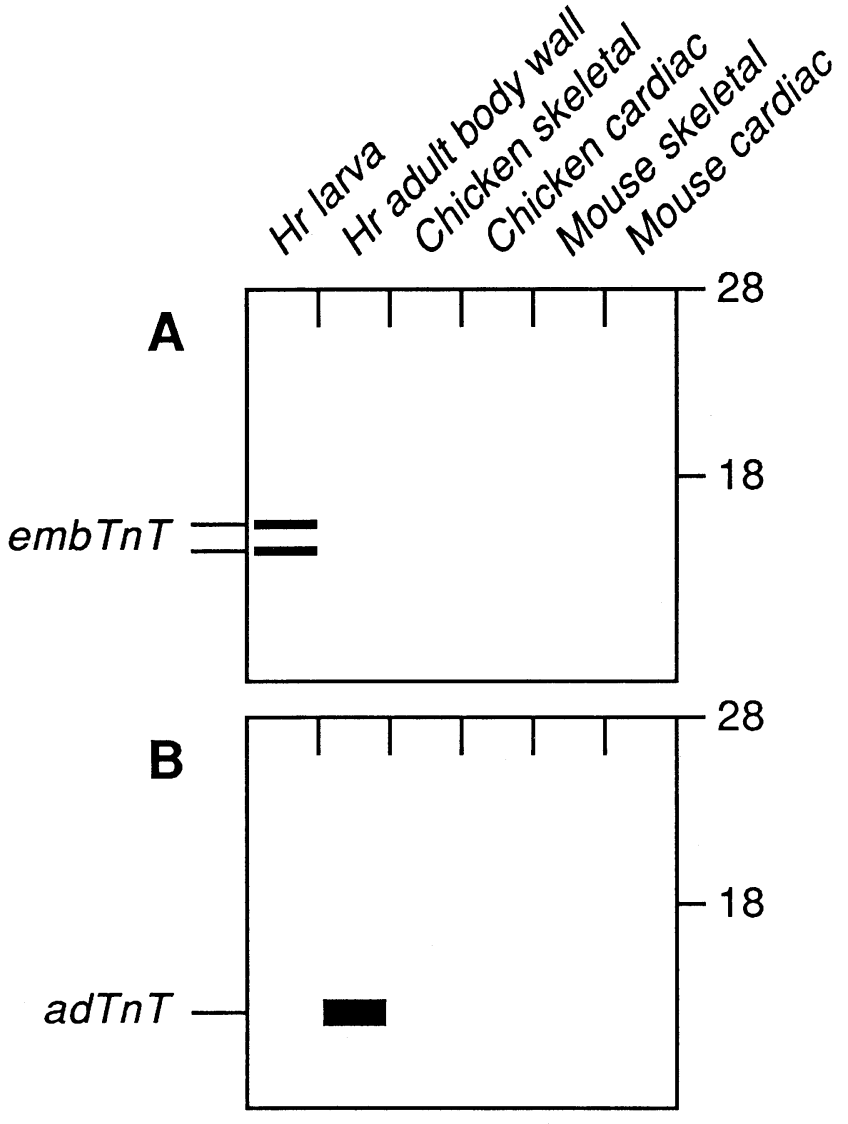

Fig. 3. Schematic drawing of Northern blots of RNAs from $H$. rore$t z i$, chicken, and mouse muscle tissues probed with eTnT11 and aTnT2. The blots were hybridized with eTnT11 (A) and with aTnT2 (B). Subjected to the blotting were RNAs from larval $H$. roretzi, adult $H$. roretzi body wall muscle, chicken skeletal and cardiac muscles, and mouse skeletal and cardiac muscles. The positions of mouse 28 and $18 \mathrm{~S}$ rRNAs are indicated.

ence in the size of the mRNA bands is close to the length between the proximal and distal putative poly(A) addition signals present in eTnT11.

Although eTnT11 was specifically expressed in the larva, it was necessary to examine whether eTnT11 was expressed in the tail striated muscle or other tissues such as heart muscle and visceral smooth muscle because poly(A) ${ }^{+}$RNA derived from whole body of the tailbud embryos was used to construct the cDNA library (22). To address this issue, we applied whole mount in situ hybridization probed with the antisense eTnT11 transcript. The hybridization was already detected in the immature tail muscle cells of the early and middle stages of the embryos. Even after the development of various organs in the late stage of the embryos and the hatched larvae, the hybridization was restricted to the striated muscle cells and excluded from heart or viscera (Fig. 4). Antisense aTnT2 transcript did not hybridize to

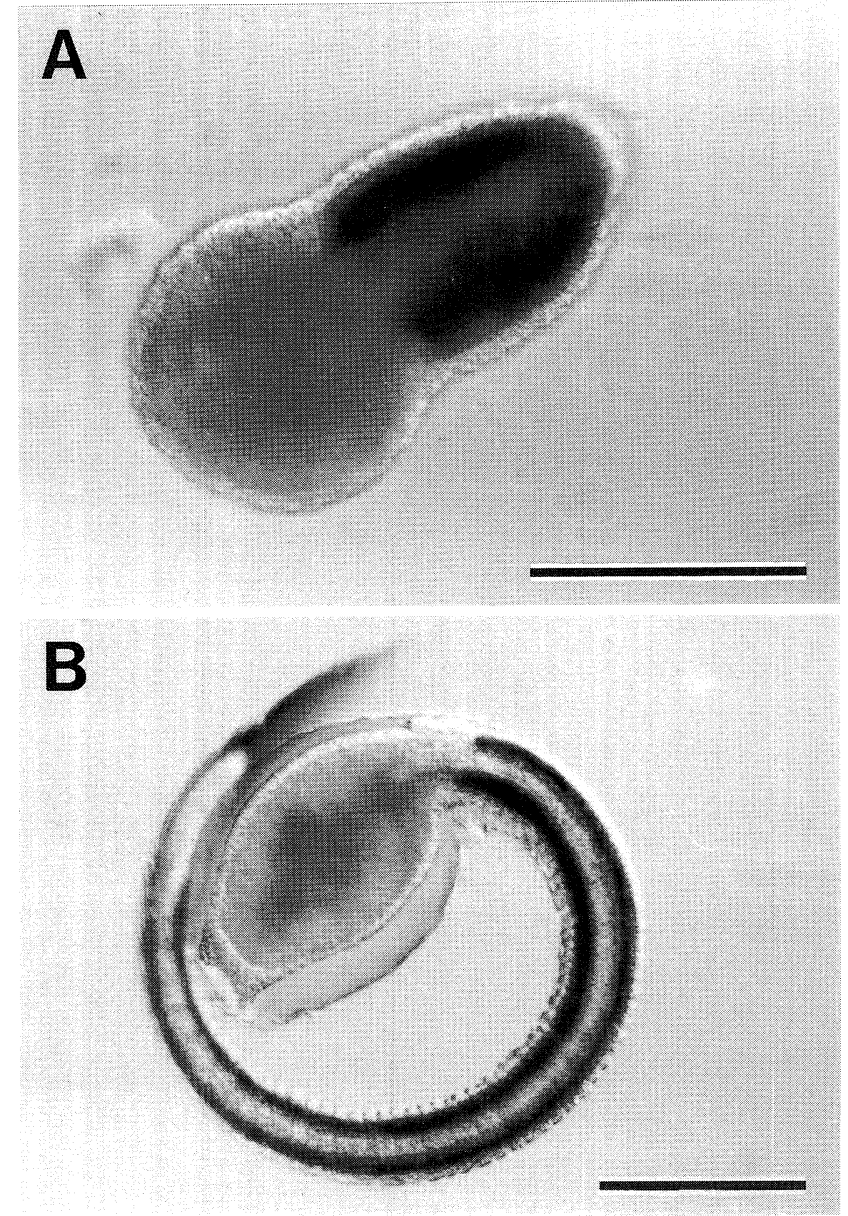

Fig. 4. Whole mount in situ hybridization of the tailbud embryos. An early (A) and a late (B) tailbud embryos were hybridized with digoxigenin-labeled antisense eTnT11 transcripts. Only the tail striated muscle cells exhibited specific staining. Scale bar, $200 \mu \mathrm{m}$ (Modified from Ref. 14).

mRNAs in any tissues of the embryos or the larvae. These results indicate that embTnT is exclusively present in the tail striated muscle at stages of the tailbud embryos and the tadpole larvae, whereas adTnT is restricted to the adult body wall muscle.

Localization of transfected $H$. roretzi TnT in mammalian fibroblasts. To investigate whether $\operatorname{adTnT}$ is able to bind to Tm in vivo, we transfected the recombinant plasmid, pSRTnT, harboring aTnT2 cDNA under the control of strong SR $\alpha$ promoter (23) to the mouse fibroblast cell line C3H10T1/2. Immunofluorescence microscopy by staining with NT302 showed that the exogenous adTnT unexpectedly accumulated in the nuclei instead of being associated with Tm-containing microfilament bundles (Fig. 5A and B). This ectopic nuclear location suggest either that adTnT has a sequence corresponding to the nuclear localization signal (NLS) or 

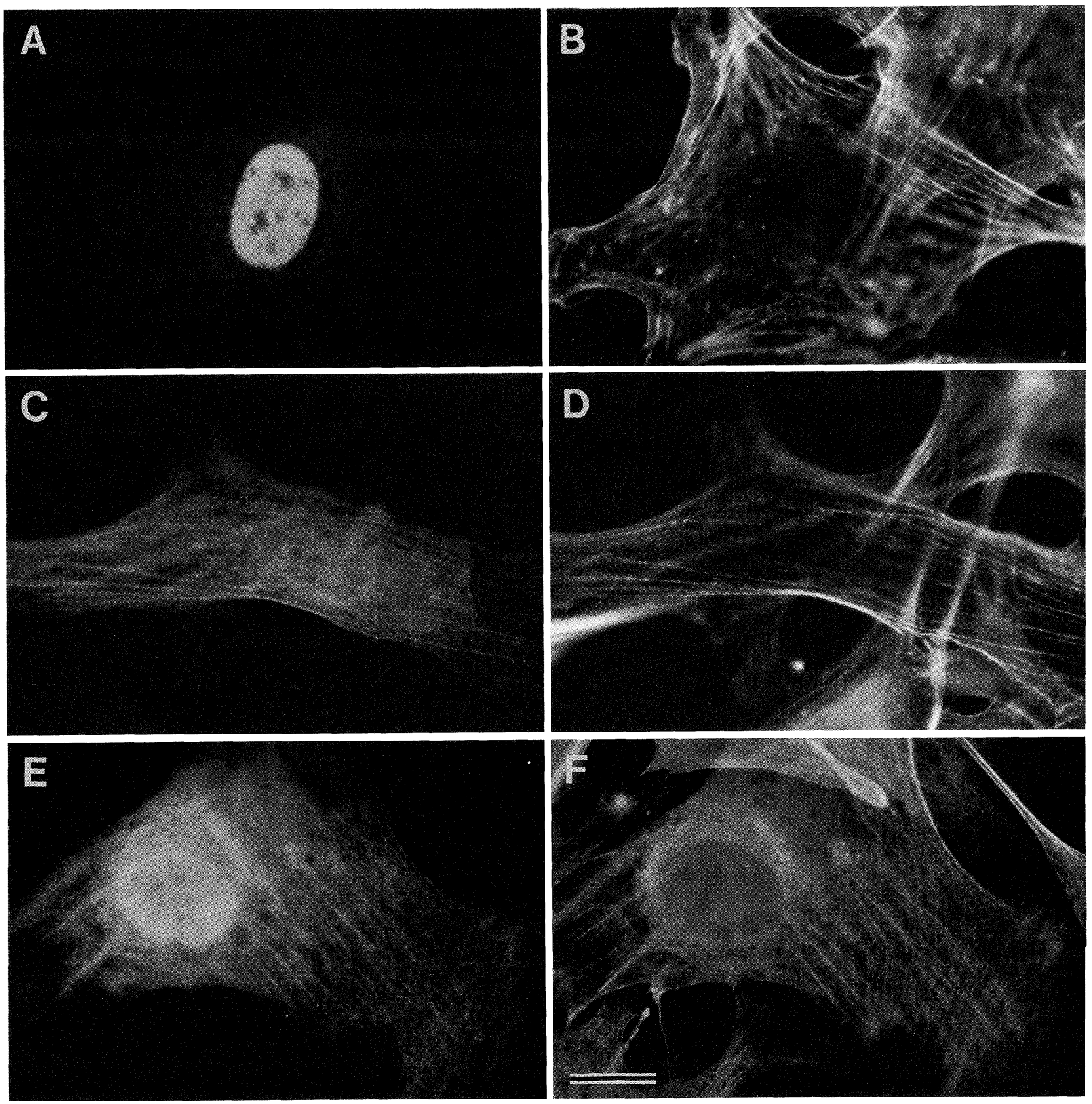

Fig. 5. Immunofluorescent localization of exogenously expressed entire and truncated adTnT. C3H10T1/2 fibroblasts were transiently transfected with pSRTnT (A and B) or pSRTnT $\triangle$ Ava (C-F). The cells were doubly stained with NT302 (A, C, and E) and rhodamine-phalloidin (B and D) or affinity-purified anti-chicken gizzard tropomyosin rabbit IgG (F). Scale bar, $20 \mu \mathrm{m}$.

that $\operatorname{adTnT}$ is imported to the nucleus in association with some nuclear protein.

We scrutinized the amino acid sequence of adTnT and noticed two clusters of basic amino acids: the $\mathrm{N}$ terminal $\left(\mathrm{R}^{107} \mathrm{~K}-\mathrm{K}^{116} \mathrm{KRK}\right)$ and the $\mathrm{C}$-terminal $\left(\mathrm{K}^{150} \mathrm{R}-\right.$ $\mathrm{K}^{157} \mathrm{KKK}$ ) clusters (Fig. 2). Since these basic amino acid clusters are similar to the NLS of the SV40 large T antigen (PKKKRKV) or the bipartite NLS of nucleoplasmin (KRPAAIKKAGQAKKKK) (24), they may have served as NLSs in the transfected cells. To confirm this postulation, we transfected the cells with the plasmid pSRTnT $\Delta$ Ava, which contains a deletion-mutated aTnT2 cDNA specifying a truncated adTnT lacking the $\mathrm{C}$-terminal cluster (Fig. 2). The truncated protein was located along the microfilament bundles detected by rhodamine-phalloidin staining (Fig. 5C and D). Double immunostaining with NT302 and anti-Tm showed that the exogenous $\mathrm{TnT}$ and endogenous $\mathrm{Tm}$ were colocalized 
on the microfilament bundles (Fig. 5E and F). Observations at a higher magnification revealed that they coexisted with a periodic distribution. These results suggest that the sequence including the $\mathrm{C}$-terminal basic amino acid cluster is responsible at least in part for the nuclear localization of adTnT and that adTnT is indeed able to interact with $\mathrm{Tm}$ in vivo when the sequence is hindered.

\section{DISCUSSION}

In the present study, we cloned two distinct TnT molecules from adult and tailbud embryo of the ascidian $H$. roretzi. This is the first case of cloning deuterostome invertebrate TnTs. Probable Tm- and TnI-binding ability of these TnTs was suggested by the existence of the conserved sequences in these TnTs corresponding to $\mathrm{Tm}$ - and TnI-binding domains in rabbit skeletal muscle $\mathrm{TnT}$. Colocalization of the truncated adTnT and endogenous nonmuscle $\mathrm{Tm}$ in the transfected cells corroborated its Tm-binding ability.

Although aTnT2 and aTnT19 were derived from distinct mRNA species, they coded for the identical protein $(\operatorname{adTnT})$. In addition, eTnT11-encoded protein (embTnT) was not detected in the adult body wall muscle. Thus, so far only a single TnT protein has been identified in the adult smooth muscle. Isoelectric focusing /SDS-PAGE analyses, however, have detected multiple isoelectric forms of body wall muscle TnT (9). At least some of these forms are likely to be generated by posttranslational modifications such as phosphorylation. In fact, there are several possible phosphorylation sites in adTnT by known protein serine/threonine kinases: $(\mathrm{S} / \mathrm{T}) \mathrm{XX}(\mathrm{D} / \mathrm{E})$ for casein kinase II (21) and (S/T)P for Cdc2 (25). S ${ }^{2}$ GEE, T ${ }^{153}$ ERE, $\mathrm{S}^{192} \mathrm{LEE}$, and $\mathrm{S}^{175} \mathrm{P}$ meet these consensus sequences. Particularly, the first site is likely to be phosphorylated by casein kinase II because this sequence is similar to $\mathrm{S}^{1} \mathrm{DEE}$, which is phosphorylatable by casein kinase (presumably casein kinase II, considering the sequence), in rabbit skeletal muscle $\mathrm{TnT}$. If various levels of phosphorylation take place at these sites, they are able to be responsible for at least some of the multiple isoelectric variants. Alternative pre-mRNA splicing generates multiple TnT mRNAs encoding separate protein isoforms in vertebrate skeletal and cardiac muscles $(16,26)$. In addition, we have to consider the possibility that multiple TnT genes are expressed in muscle tissue. It remains to be determined which of these three mechanisms governs the production of multiple isoelectric forms of body wall muscle TnT.

Striated and smooth muscles in higher vertebrates usually express their tissue type-specific isoforms of muscle proteins. In addition, transition of developmental stage-specific isoforms often occurs. In the case of $H$. roretzi, discrete TnT cDNAs were cloned from the tailbud embryo and the adult body wall muscle. Northern blotting and in situ hybridization further showed that embTnT mRNA was specifically present in the embryonic/larval tail striated muscle but absent from the adult smooth muscle. In contrast, adTnT mRNA was detected in the adult muscle but not at the larval stage. In another ascidian $C$. intestinalis, one major isoform of $\mathrm{Tm}$ expressed in the adult body wall muscle is also present in striated muscles of heart and larval tail (18). If a single species of Tm persists throughout the development of $H$. roretzi as in $C$. intestinalis, the temporospatial expression pattern of $\mathrm{Tm}$ is different from that of TnT. Considering the difference in contraction rate between the embryonic/larval tail muscle and the adult body wall muscle, Tns in these muscles would differentially regulate the contraction, i.e., $\mathrm{Tn}$ in the tail muscle might be a $\mathrm{Ca}^{2+}$-dependent repressor like vertebrate $\mathrm{Tn}$, whereas $\mathrm{Tn}$ in the adult body wall muscle is a $\mathrm{Ca}^{2+}$-dependent activator. The difference in the regulation would be ascribable at least in part to the functional difference between embTnT and adTnT. Nevertheless, both types of TnTs are likely to interact with the same species of $\mathrm{Tm}$, because adTnT seems to interact even with the mouse fibroblast Tm. These postulations may explain the different temporospatial expression pattern of Tm and TnT.

Despite the thin filament-associated localization of $H$. roretzi $\mathrm{Tn}$ in the body wall muscle (6), the exogenously expressed entire length of adTnT was mainly located in the nuclei of the mouse fibroblasts. Deletion of the sequence including the $\mathrm{C}$-terminal basic amino acid cluster suggested that this region functioned as the NLS. If $\mathrm{TnI}$ and $\mathrm{TnC}$ are synthesized concurrently with the intact TnT in vivo, however, prompt complex formation may occur. Association of the Tn complex with thin filament-linked $\mathrm{Tm}$ in vivo suggests that the complex formation results in concealment of the NLS. Hence we have to be careful when we analyze the localization and effects of certain proteins by introducing the proteins or their cDNAs into cells. This is particularly applicable to subunit proteins that comprise a complex molecule.

Acknowledgments. We are grateful to Drs. Kazuhiro W. Makabe and Noriyuki Satoh for their generous gift of the tailbud embryo cDNA library. This study was supported by Grants-in-Aid for Scientific Research from the Ministry of Education, Science, Sports and Culture of Japan.

\section{REFERENCES}

1. Ebashi, S. 1978. The regulatory role of calcium in muscle. Ann. N.Y. Acad Sci., 307: 451-461.

2. KaMm, K.E. and Stull, J.T. 1985. The function of myosin light chain kinase phosphorylation in smooth muscle. Annu. Rev. Pharmacol. Toxicol., 25: 593-620.

3. SelLers, J.R. 1991. Regulation of cytoplasmic and smooth 
muscle myosin. Curr. Opin. Cell Biol., 3: 98-104.

4. Lehman, W. and Szent-Györgi, A.G. 1975. Regulation of muscular contraction. Distribution of actin control and myosin control in the animal Kingdom. J. Gen. Physiol., 66: 1-30.

5. Toyota, N., Obinata, T., and Terakado, K. 1979. Isolation of troponin-tropomyosin-containing thin filaments from ascidian smooth muscle. Comp. Biochem. Physiol., 62B: 433-441.

6. Endo, T. and Obinata, T. 1981. Troponin and its components from ascidian smooth muscle. J. Biochem., 89: 15991608.

7. TeraKado, K. and Obinata, T. 1987 . Structure of multinucleated smooth muscle cells of the ascidian Halocynthia roretzi. Cell Tissue Res., 247: 85-94.

8. Obinata, T., Ooi, A., and Takano-Ohmuro, H. 1983. Myosin and actin from ascidian smooth muscle and their interaction. Comp. Biochem. Physiol., 76B: 437-442.

9. Ohshima, S., KomiYa, T., Takeuchi, K., Endo, T., and Obinata, T. 1988. Generation of multiple troponin T isoforms is a common feature of the muscles in various chordate animals. Comp. Biochem. Physiol., 90B: 779-874.

10. Аве, Н., Комiya, Т., and Obinata, T. 1986. Expression of multiple troponin $\mathrm{T}$ variants in neonatal chicken breast muscle. Dev. Biol., 118: 42-51.

11. Makabe, K.W., Fujiwara, S., Saiga, H., and Satoh, N. 1990. Specific expression of myosin heavy chain gene in muscle lineage cells of the ascidian embryo. Roux's Arch. Dev. Biol., 199: 307-313.

12. Kusakabe, T., Suzuki, J., Saiga, H., Jeffery, W.R., MaKabe, K.W., and SАтоH, N. 1991. Temporal and spatial expression of a muscle actin gene during embryogenesis of the ascidian $\mathrm{Hal}$ ocynthia roretiz. Dev. Growth Differ., 33: 227-234.

13. MeEdel, T.H. and Hastings, K.E.M. 1993. Striated muscletype tropomyosin in a chordate smooth muscle, ascidian bodywall muscle. J. Biol. Chem., 268: 6755-6564.

14. Endo, T., Matsumoto, K., Hama, T., Ohtsuka, Y., Katsura, G., and OBinata, T. 1996. Distinct troponin T genes are expressed in embryonic/larval tail striated muscle and adult body wall smooth muscle of ascidian. J. Biol. Chem., 271: 2785527862 .

15. Smillie, L.B., Golosinska, K., and Reinach, F.C. 1988. Sequences of complete cDNAs encoding four variants of chicken skeletal muscle troponin T. J. Biol. Chem., 263: 18816-18820.

16. COOPER, T.A. and ORDAHL, C.P. 1985 . A single cardiac tropo$\operatorname{nin} \mathrm{T}$ gene generates embryonic and adult isoforms via developmentally regulated alternate splicing. J. Biol. Chem., 260: 11140-11148.

17. Pearlstone, J.R., Johnson, P., Carpenter, M.R., and SMILLIE, L.B. 1977. Primary structure of rabbit skeletal muscle troponin-T. Sequence determination of the $\mathrm{NH}_{2}$-terminal fragment CB3 and the complete sequence of troponin-T. J. Biol. Chem., 252: 983-989.

18. Pearlstone, J.R., Carpenter, M.R., and Smillie, L.B. 1986. Amino acid sequence of rabbit cardiac troponin T. $J$. Biol. Chem., 261: 16795-16810.

19. Ohtsuki, I. and Nagano, K. 1982. Molecular arrangement of troponin-tropomyosin in the thin filament. Adv. Biophys., 15: 93-130.

20. Lipman, D.J. and Pearson, W.R. 1985. Rapid and sensitive protein similarity searches. Science, 227: 1435-1441.

21. Krebs, E.G., Eisenman, R.N., Kuenzel, E.A., Litchfield, D.W., Lozeman, F.J., LÜscher, B., and Sommercorn, J. 1988. Casein kinase II as a potentially important enzyme concerned with signal transduction. Cold Spring Harbor Symp. Quant. Biol., 53: 77-84.

22. MaKABE, K.W. and SAтон, N. 1989. Temporal expression of myosin heavy chain gene during ascidian embryogenesis. Dev. Growth Differ., 31: 71-77.

23. Takebe, Y., Seiki, M., Fujisawa, J., Hoy, P., Yokota, K., Arai, Y., Yoshida, M., and ARAI, N. 1988. SR $\alpha$ promoter: an efficient and versatile mammalian cDNA expression system composed of the simian virus 40 early promoter and the R-U5 segment of human T-cell leukemia virus type 1 long terminal repeat. Mol. Cell Biol., 8: 466-472.

24. Dingwall, C. and Laskey, R.A. 1991. Nuclear targeting sequences - a consensus? Trends Biochem. Sci., 16: 478-481.

25. NiGG, E.A. 1993. Targets of cyclin-dependent protein kinases. Curr. Opin. Cell Biol., 5: 187-193.

26. Breitbart, R.E., Nguyen, H.T., Medford, R.M., Destree, A.T., Mahdavi, V., and Nadal-Ginard, B. 1985. Intricate combinatorial patterns of exon splicing generate multiple regulated troponin $\mathrm{T}$ isoforms from a single gene. Cell, 41: 67-82. 